(2) Tsubomura, H.; Mulliken, R. S. J. Am. Chem. Soc. 1960, 82, 5966.

(3) Quan, N. N.; Guzzo, A. V. J. Phys. Chem. 1981, 85, 2303.

(4) Razi Naqui, K. J. Phys. Chem. 1981, 85, 2303.

(5) Blättler, C.; Jent, F.; Paul, H. Chem. Phys. Lett. 1990, 166, 375.

(6) Kawai, A.; Okutsu, T.; Obi, K. J. Phys. Chem. 1991, 95, 9130.

(7) Kawai, A.; Obi, K. J. Phys. Chem. 1992, 96, 52.

(8) Imamura, T.; Onitsuka, O.; Obi, K. J. Phys. Chem. 1986, 90, 6741.

(9) Adrian, F. J. Rev. Chem. Intermed. 1979, 3, 3. Muss, L. T.; Atkins, P. W.; McLauchlan, K. A.; Pedersen, J. B. Chemically Induced Magnetic Polarization; Reidel: Dordrecht, 1977.
(10) Trifunac, A. D. Chem. Phys. Lett. 1977, 49, 457.

(11) Murai, H.; Imamura, T.; Obi, K. Chem. Phys. Lett. 1982, 87, 295.

(12) Murov, S. L. Handbook of Photochemistry; Marcel Dekker, Inc. New York, 1973.

(13) Kivelson, D. J. Chem. Phys. 1960, 33, 1094.

(14) Turro, N. J. Modern Molecular Photochemistry; Benjamin/Cummings: Reading, MA, 1978.

(15) Adrian, F. J.; Monchick, L. J. Chem. Phys. 1979, 7l, 2600; J. Chem. Phys. 1980, 72, 5786.

(16) Pedersen, J. B.; Freed, J. H. J. Chem. Phys. 1973, 58, 2746.

\title{
Exponentiating Trajectories on a Realistic Potential Energy Surface for $\mathrm{Na}_{3}$
}

\author{
V. M. F. Morais ${ }^{\dagger}$ and A. J. C. Varandas* $*$ \\ Instituto de Ciências Biomédicas Abel Salazar, Universidade do Porto, 4000 Porto, and Centro de Quimica, \\ Departamento de Quimica, Universidade de Coimbra, 3049 Coimbra Codex, Portugal, and Departamento de \\ Quimica, Universidade de Coimbra, 3049 Coimbra Codex, Portugal (Received: December 9, 1991)
}

\begin{abstract}
A classical intramolecular dynamics study for the ground electronic state of the sodium trimer has been carried out using a realistic potential energy surface previously reported by the authors. It is shown that the trajectories are exponentially diverging even for very low energies close to the bottom of the potential well. This fact is confirmed by the calculated maximum Lyapunov characteristic numbers, which suggest that the $\mathrm{Na}_{3}$ molecule possesses a small vibrational relaxation time. This result corroborates previous findings on atom-diatom reactive scattering for analogous systems, which have indicated a high degree of statistical behavior in the dynamics of these reactions.
\end{abstract}

\section{Introduction}

As it is well-known, a long-standing problem in equilibrium statistical mechanics is the justification of the assumption of equipartition of the energy, which plays a central role in several statistical theories of unimolecular reactions (such as the RRKM theory ${ }^{1,2}$ ). In fact, those statistical theories contain assumptions connected with the stochastic motion of molecules at their dissociation energies. It is therefore important to understand the mechanisms that govern the transition to stochastic motion, as well as the rate of energy randomization between the molecular degrees of freedom.

In the past few decades there has been great progress in the theoretical study of dynamical systems which is relevant for the understanding of the transition from dynamics to statistical mechanics. In particular, several numerical experiments have been performed in order to verify the validity of the assumptions accepted by classical statistical mechanics. Unfortunately the vast majority of those numerical calculations refer to simple model systems with few degrees of freedom.

Those advances have shown that the classical motion of a bound system with $N$ degrees of freedom, governed by a nonseparable potential, can fall into several different patterns. At low energies, and for approximately integrable potentials, the dynamical system is completely integrable and its trajectories are constrained to an $N$ dimensional hypersurface in the $2 N$ dimensional phase space, called an invariant toroid, which is completely defined by the values of the $N$ isolating integrals of motion. This behavior is usually termed quasiperiodic motion and can be characterized by two major features: (1) the power spectra of dynamical variables are discrete and vary sharp and (2) initially adjacent trajectories deviate linearly with time.

On the other hand, at high energies or large anharmonicities, the number of isolating integrals of motion is drastically reduced. For nonrotating systems the only isolating integral of motion is then the total energy and its trajectory tends to cover (after a possibly long time) the whole $2 N-1$ dimensional energy shell.

\footnotetext{
Universidade do Porto and Centro de Quimica, Departamento de Quimica, Universidade de Coimbra.
}

'Departamento de Quimica, Universidade de Coimbra.
In this regime the system is said to have an ergodic or chaotic behavior.

The power spectra of dynamical variables are now almost continuous and trajectories initially in close proximity tend to deviate exponentially in such a way that they become completely uncorrelated, which accounts for the mixing properties of the system. ${ }^{3,4}$ Stochastic trajectories in ergodic theory have been considered as the main cause for statistical behavior. ${ }^{3,5}$

The inverse of the rate of exponential divergence has been interpreted as the time that the system needs to achieve a microcanonical distribution (or better, as the inverse decay time of correlations) ${ }^{6,7}$ This rate of divergence is the maximal Lyapunov characteristic number and it has been proved that it is related to the Kolmogorov entropy. 8,9

The onset of chaotic behavior in dynamical systems is of great importance because of its connection with the possibility of occurrence of rapid relaxation of the internal energy among its degrees of freedom. Numerical studies have shown that the transition from predominantly regular to predominantly irregular behavior is usually rather abrupt. ${ }^{10}$ Nevertheless, numerical calculations by Contopoulos et al. ${ }^{11}$ for a system of three degrees of freedom with a galactic type potential have shown that stochastic and quasiperiodic trajectories can exist at the same energy. In this case two regions of phase space with different stochastic behavior could be distinguished on the basis of the maximal Lyapunov characteristic numbers: one which shows the existence of an isolating integral of motion beyond the energy and the other with the energy as the only constant of the motion. It can therefore be concluded that microscopically small regions of instability may exist at low energies, and, conversely, it may happen that a small fraction of phase space is occupied by quasiperiodic trajectories at high energies. ${ }^{10,12}$

Studying the classical dynamics of a molecule requires an analytical function for the potential energy surface. The characteristics of the dynamics clearly depend entirely on such functions (for example, the two-dimensional Toda potential, 13,14 although anharmonic, gives a completely integrable system for all energies up to infinity). In this paper we report a classical intramolecular dynamics study for the electronic ground doublet state of $\mathrm{Na}_{3}$ using a realistic potential energy surface which has 
been described elsewhere. ${ }^{15}$ The present calculations are based on the premise that only the lowest sheet of this ground doublet state potential energy surface is necessary. Thus, no allowance is made for the hopping of trajectories from this sheet to the upper one, which forms a degenerate pair with the former at $D_{3 h}$ geometries.

The paper is organized as follows. In the next section we describe the equations of motion and computational methods used. The details of the potential energy surface used for $\mathrm{Na}_{3}$ are then briefly reviewed in section 3 . Section 4 contains the results while some conclusions are gathered in section 5 where we also compare our results with those from previous work. We examine only the rotationless molecule. Accordingly, we are dealing with a system having three degrees of freedom.

\section{Equations of Motion and Computational Methods}

Let us consider a nonrotating triatomic molecule $\mathrm{ABC}$, and let $m_{\mathrm{A}}, m_{\mathrm{B}}$, and $m_{\mathrm{C}}$ be the masses of atoms $\mathrm{A}, \mathrm{B}$, and $\mathrm{C}$, respectively. Neglecting the (uniform) motion of the center of mass, the Hamiltonian for such a system can be written as ${ }^{16,17}$

$$
\begin{aligned}
& H=\frac{m_{\mathrm{A}}+m_{\mathrm{C}}}{2 m_{\mathrm{A}} m_{\mathrm{C}}} P_{1}^{2}+\frac{m_{\mathrm{B}}+m_{\mathrm{C}}}{2 m_{\mathrm{B}} m_{\mathrm{C}}} P_{2}^{2}+\frac{\cos Q_{3}}{m_{\mathrm{C}}} P_{1} P_{2}+ \\
& \frac{A}{2 m_{\mathrm{A}} m_{\mathrm{B}} m_{\mathrm{C}} Q_{1}^{2} Q_{2}^{2}} P_{3}^{2}-\frac{\sin Q_{3}}{m_{\mathrm{C}}} P_{3}\left(\frac{P_{1}}{Q_{2}}+\frac{P_{2}}{Q_{1}}\right)+V\left(Q_{1}, Q_{2}, Q_{3}\right)
\end{aligned}
$$

where $Q_{1}$ and $Q_{2}$ are the interatomic distances $\mathrm{A}-\mathrm{C}$ and $\mathrm{B}-\mathrm{C}$, respectively, $Q_{3}$ is the included angle and $P_{1}, P_{2}$, and $P_{3}$ are the momenta conjugate to canonical coordinates $Q_{1}, Q_{2}$, and $Q_{3}$. In the above equation $A$ represents a numerical factor defined as $A=$

$$
m_{\mathrm{A}}\left(m_{\mathrm{B}}+m_{\mathrm{C}}\right) Q_{1}{ }^{2}+m_{\mathrm{B}}\left(m_{\mathrm{A}}+m_{\mathrm{C}}\right) Q_{2}^{2}-2 m_{\mathrm{A}} m_{\mathrm{B}} Q_{1} Q_{2} \cos Q_{3}
$$

The Hamiltonian equations of motion for this system are then given by

$$
\begin{gathered}
\frac{\partial P_{1}}{\partial t}=\frac{\left(m_{\mathrm{A}}+m_{\mathrm{C}}\right) Q_{2}-m_{\mathrm{A}} Q_{1} \cos Q_{3}}{m_{\mathrm{A}} m_{\mathrm{C}} Q_{1}{ }^{3} Q_{2}} P_{3}^{2}-\frac{\sin Q_{3}}{m_{\mathrm{C}} Q_{1}{ }^{2}} P_{2} P_{3}-V_{1} \\
\frac{\partial P_{2}}{\partial t}=\frac{\left(m_{\mathrm{B}}+m_{\mathrm{C}}\right) Q_{1}-m_{\mathrm{B}} Q_{2} \cos Q_{3}}{m_{\mathrm{B}} m_{\mathrm{C}} Q_{1} Q_{2}{ }^{3}} P_{3}^{2}-\frac{\sin Q_{3}}{m_{\mathrm{C}} Q_{2}{ }^{2}} P_{1} P_{3}-V_{2} \\
\frac{\partial P_{3}}{\partial t}=\left(m_{\mathrm{C}} Q_{1} Q_{2}\right)^{-1}\left[Q_{1} Q_{2} P_{1} P_{2}+P_{3}^{2} \sin Q_{3}-\left(Q_{1} P_{1}+\right.\right. \\
\frac{\partial Q_{1}}{\partial t}=\frac{m_{\mathrm{A}}+m_{\mathrm{C}}}{m_{\mathrm{A}} m_{\mathrm{C}}} P_{1}+\frac{\cos Q_{3}}{m_{\mathrm{C}}} P_{2}-\frac{\sin Q_{3}}{m_{\mathrm{C}} Q_{2}} P_{3} \\
\frac{\partial Q_{2}}{\partial t}=\frac{\cos Q_{3}}{m_{\mathrm{C}}} P_{1}+\frac{m_{\mathrm{B}}+m_{\mathrm{C}}}{m_{\mathrm{B}} m_{\mathrm{C}}} P_{2}-\frac{\sin Q_{3}}{m_{\mathrm{C}} Q_{1}} P_{3} \\
\frac{\partial Q_{3}}{\partial t}=-\frac{\sin Q_{3}}{m_{\mathrm{C}} Q_{1}} P_{2}+\left(\frac{m_{3}}{m_{\mathrm{A}} m_{\mathrm{B}} m_{\mathrm{C}} Q_{1}{ }^{2} Q_{2}{ }^{2}}-\frac{\sin Q_{3}}{m_{\mathrm{C}} Q_{2}}\right) P_{3}
\end{gathered}
$$

where the notation $V_{i}(i=1,2,3)$ stands for the first partial derivative of the potential energy surface with respect to the coordinate $Q_{i}$.

In order to solve the dynamical problem one needs to specify the initial conditions for the system of differential equations 3-8. To achieve this goal in a straightforward manner the concept of normal modes reveals great utility. It must be noted however that normal mode descriptions are strictly valid only for purely quadratic (harmonic) hamiltonians.

The normal mode analysis may then be carried out using the Wilson $F G$ method ${ }^{18,19}$ yielding the transformation matrix, $A$, from internal $\left(Q_{1}, Q_{2}, Q_{3}\right)$ to normal mass scale coordinates $\left(\xi_{1}, \xi_{2}, \xi_{3}\right)$ and its inverse

$$
\boldsymbol{\xi}=\mathbf{A Q}
$$

where, as usual, the boldface notation identifies matrix variables. These transformations also allow the transformation between internal $\left(P_{1}, P_{2}, P_{3}\right)$ and normal $\left(\Pi_{1}, \Pi_{2}, \Pi_{3}\right)$ momenta. The initial conditions are next chosen such that the molecule always starts its motion at the minimum of the potential energy surface, which in this case has the geometry of an isosceles triangle with $Q_{1}=$ $Q_{2}=6.12 a_{0}$ and an "obtuse" included angle of $Q_{3}=101.35^{\circ}$, corresponding to the electronic state ${ }^{2} B_{2}$. (Unless mentioned otherwise, atomic units were used in this work: au of length, $a_{0}$ $=0.529177 \times 10^{-10} \mathrm{~m}$; au of energy, $E_{\mathrm{h}}=2625.47 \mathrm{~kJ} \mathrm{~mol}^{-1}$; au of time $(\mathrm{atu})=2.419 \times 10^{-17} \mathrm{~s}$.) With this choice of the initial configuration, one obtains for the initial values of the normal coordinates

$$
\xi_{1}=\xi_{2}=\xi_{3}=0
$$

The energy of the molecule in the vibrational quantum state with quantum numbers $\left(n_{1}, n_{2}, n_{3}\right)$ may then be written (referred to the minimum of the potential energy surface, which is taken as the zero of energy) as

$$
E=\sum_{i=1}^{3} h v_{i}\left(n_{i}+1 / 2\right)
$$

or, classically, as

$$
E=\sum_{i=1}^{3} 1 / 2\left(\Pi_{i}^{2}+4 \pi^{2} v_{i}^{2} \xi_{i}^{2}\right)
$$

where $v_{i}=c w_{i}, w_{i}(i=1,2,3)$ are the harmonic frequencies in units of $\mathrm{cm}^{-1}$, and $c$ is the speed of the light in the vacuum. At the minimum of the potential energy surface one then gets from eq 10 to eq 12

$$
E_{i}=h v_{i}\left(n_{i}+1 / 2\right)=1 / 2 \Pi_{i}^{2}
$$

or, solving for the normal momenta

$$
\Pi_{i}= \pm\left[2 h v_{i}\left(n_{i}+1 / 2\right)\right]^{1 / 2}
$$

Thus, eqs 13 and 14 allow us to specify the initial values of the normal momenta.

The next step required to initiate the integration of the Hamilton equations, eqs 3-8, is then to obtain the initial values of the internal momenta, through the inverse of transformation of eq 9 . The integration of the Hamilton equations may now be carried out numerically using any of the available numerical integration algorithms.

\section{Potential Energy Surface}

The $\mathrm{Na}_{3}$ potential energy surface used for the calculations of the present work is of the extended-LEPS form described elsewhere, ${ }^{15}$ the reader being referred to the original paper for details. It suffices to mention here that we use the potential energy surface associated to the parameter value $\gamma=2.5$ defined in ref 15 . The reason for this choice is that the potential energy surface so defined has a somewhat larger energy separation between the most relevant topographical features and hence is expected to be best suited for displaying a transition from quasiperiodic to chaotic motion. Such topographical details are clearly seen from Figure 1, which shows a relaxed triangular $\operatorname{plot}^{20}$ of the $\mathrm{Na}_{3}$ potential energy surface used in the present work. To obtain this plot, the perimeter of the triangle formed by the three atoms has been relaxed so as to give the lowest potential energy at each value of the geometry defined by $\left(Q_{2}{ }^{*}, Q_{3}{ }^{*}\right)$. A total of 1501 perimeters (which were obtained by varying $P$ between 0.56 and $75.56 a_{0}$ at intervals of $0.05 a_{0}$ ) were sampled. As shown in Figure 1, this $\mathrm{Na}_{3}$ potential energy surface predicts the absolute minima to have an isosceles "obtuse" triangle geometry $\left({ }^{2} B_{2}\right)$, with equal sides of $6.1247 a_{0}$, and an included angle of $101.35^{\circ}$. The other critical points of the $\mathrm{Na}_{3}$ potential energy function shown in Figure 1 are three equivalent saddle points connecting the ${ }^{2} \mathrm{~B}_{2}$ minima, corresponding to isosceles triangles $\left({ }^{2} A_{1}\right)$ with sides equal to $7.3480 a_{0}$ and an included angle of $46.64^{\circ}$, 


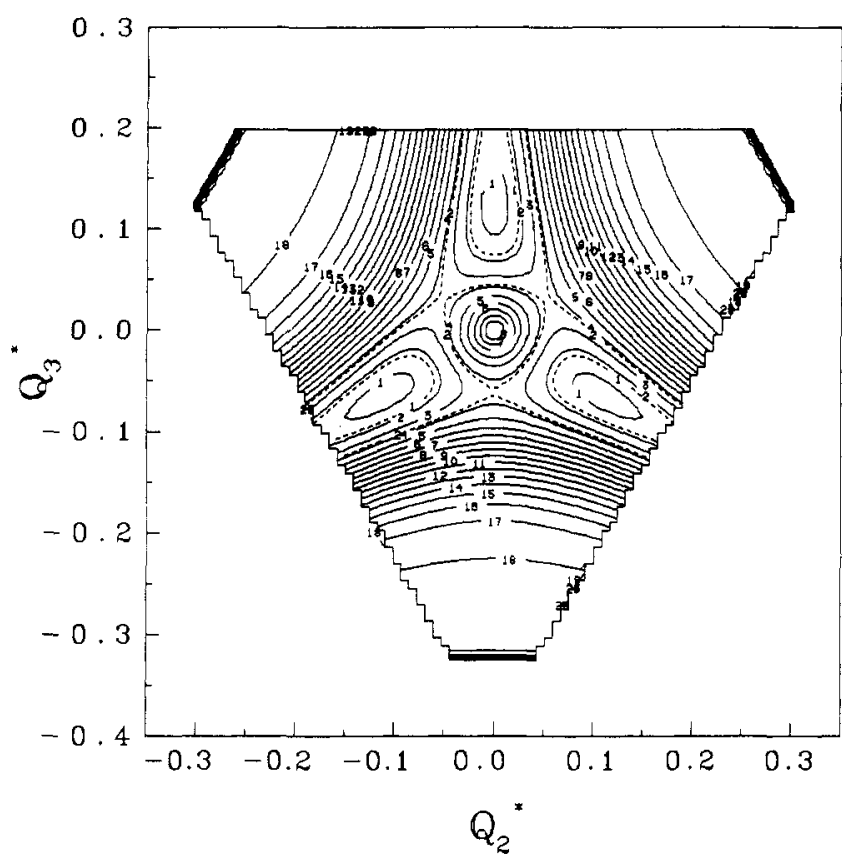

Figure 1. Relaxed triangular plot $^{20}$ for the $\mathrm{Na}_{3}$ potential energy surface used in the present work. Contours are equally spaced by $1.5 \mathrm{~kJ} \mathrm{~mol}^{-1}$, starting at $-26 \mathrm{~kJ} \mathrm{~mol}^{-1}$. The special contours shown by the dashed lines indicate the energies associated to the $(0,0,0)$ and $(1,1,1)$ vibrational levels, being numbered 1 and 2 , respectively. $Q_{2}^{*}=\left(s_{2}-s_{3}\right) / \sqrt{2}, Q_{3}^{*}$ $=\left(2 s_{1}-s_{2}-s_{3}\right) / \sqrt{6}, s_{i}=R_{i} / \sum_{j=1}^{3} R_{j}(i=1-3)$.

and three other equivalent saddle points (located at the middle point on the sides of the triangle in Figure 1) that correspond to symmetric linear species $\left({ }^{2} \Sigma_{u}{ }^{+}\right)$with sides equal to $6.1066 a_{0}$. Also shown at the center of the triangular plot is the minimum energy structure along the $D_{3 h}$ line corresponding to the conical intersection between the lower and upper sheets of the $\mathrm{Na}_{3}$ potential energy surface. Such structure has the shape of an equilateral triangle $\left({ }^{2} \mathrm{E}^{\prime}\right)$ with sides equal to $6.5653 a_{0}$. The energies of these various configurations relative to the atom-diatom asymptote are $\left(-1.0106 \times 10^{-2}\right) E_{\mathrm{h}}\left(-26.5 \mathrm{~kJ} \mathrm{~mol}^{-1}\right)$ for the ${ }^{2} \mathrm{~B}_{2}$ minimum, $\left(-8.3745 \times 10^{-3}\right) E_{\mathrm{h}}\left(-22.0 \mathrm{~kJ} \mathrm{~mol}^{-1}\right)$ for the ${ }^{2} \mathrm{~A}_{1}$ saddle point, $\left(-9.7254 \times 10^{-3}\right) E_{\mathrm{h}}\left(-25.5 \mathrm{~kJ} \mathrm{~mol}^{-1}\right)$ for the ${ }^{2} \Sigma_{\mathrm{u}}^{+}$saddle point, and $\left(-4.1801 \times 10^{-3}\right) E_{\mathrm{h}}\left(-11.0 \mathrm{~kJ} \mathrm{~mol}^{-1}\right)$ for the ${ }^{2} \mathrm{E}^{\prime}$ species. The harmonic normal frequencies for the ${ }^{2} \mathrm{~B}_{2}$ species are 130 (symmetric stretching), 106 (asymmetric stretching), and $21 \mathrm{~cm}^{-1}$ (bending).

\section{Results}

For the present investigation on the nature of the intramolecular dynamics for the sodium trimer we followed two different methodologies. First a global analysis involving the integration of some trajectories, and the subsequent calculation of the power spectra for selected dynamical variables, has been made. Then we performed a more local analysis leading to the evaluation of the rate of divergence of initially adjacent trajectories, which is measured by the so-called maximal Lyapunov characteristic number.

4.1. Spectral Analysis. Trajectories, with initial conditions specified as above, were integrated at several energies using an Adams-Moulton eleventh-order predictor-corrector algorithm initialized by a Runge-Kutta fourth-order method ${ }^{21}$ with a time step of $10^{-15} \mathrm{~s}$. Due to the well-known flat nature of the potential energy surfaces for the alkali metal trimers, ${ }^{15,22}$ only states with very low kinetic energies have been considered in the current study, namely $1.54,2.156$, and $3.08 \mathrm{~kJ} \mathrm{~mol}^{-1}$ that are associated to the classical vibrational states $\left(n_{1}, n_{2}, n_{3}\right)=(0,0,0),(0.2,0.2,0.2)$, and $(0.5,0.5,0.5)$, respectively. The numerical integration has been carried out in internal coordinates, with some periodical sampling of the calculated trajectories being done to obtain the normal coordinates and momenta. The frequency of this sampling, together with the total time for the integration, are the critical factors
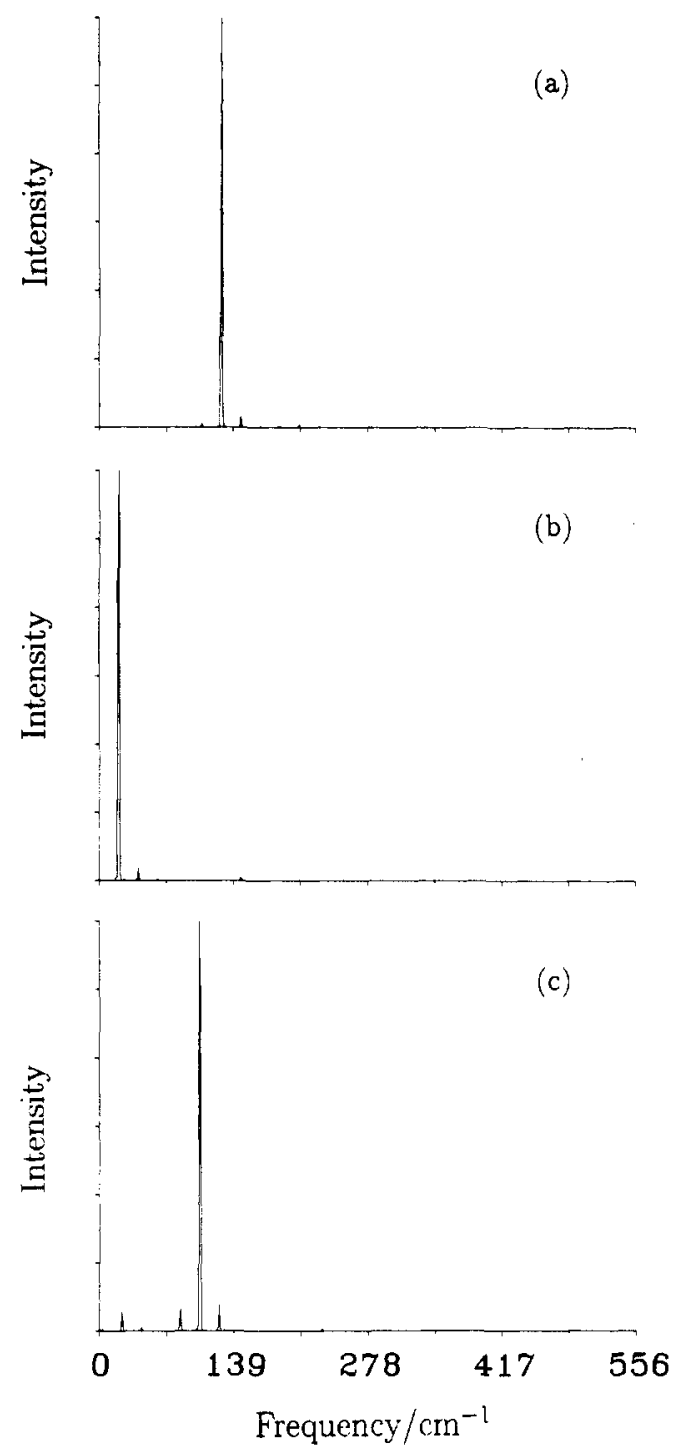

Figure 2. Power spectra for the $(0,0,0)$ vibrational state of $\mathrm{Na}_{3}$ of (a) $\Pi_{1} ;$ (b) $\Pi_{2} ;$ (c) $\xi_{3}$.

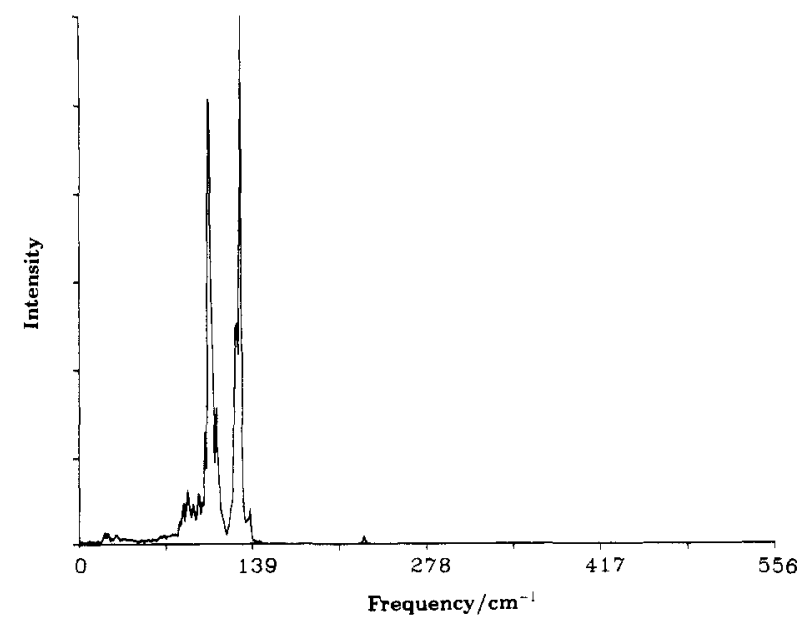

Figure 3. Power spectrum of the $\xi_{3}$ normal coordinate of $\mathrm{Na}_{3}$ for a vibrational state defined by $(0.2,0.2,0.2)$; see the text.

that determine the resolution of the resulting Fourier spectrum. For the present work we have chosen to integrate the trajectories for a time of $245.73 \mathrm{ps}$, and performed the above sampling at 8192 $\left(2^{13}\right)$ evenly spaced occasions. The power spectrum could then be estimated at 512 frequency values. The fast Fourier transform analysis was carried out using the IMSL routine FTFPS..$^{23,24}$

Shown in Figures 2-4 are the power spectra calculated at the above mentioned energies. These results are consistent with the 


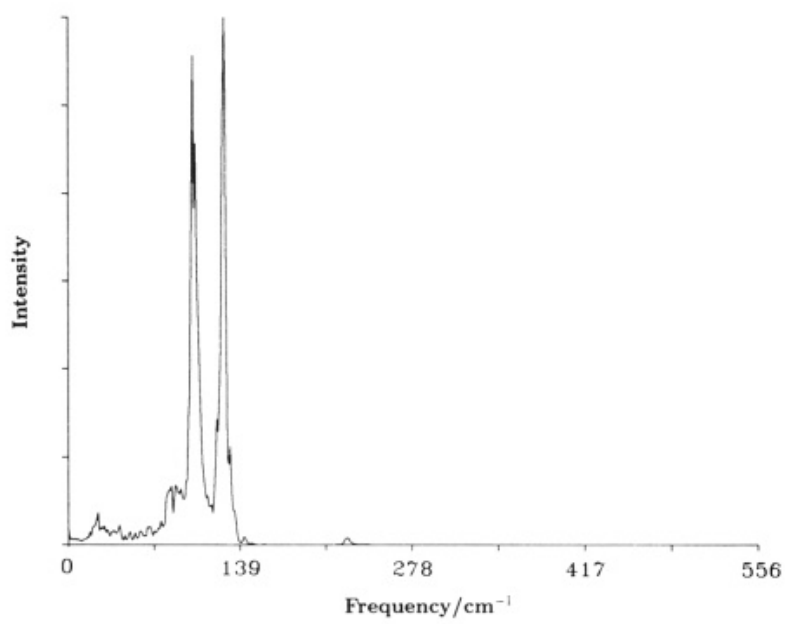

Figure 4. Same as in Figure 3 but for the vibrational state $(0.5,0.5,0.5)$.

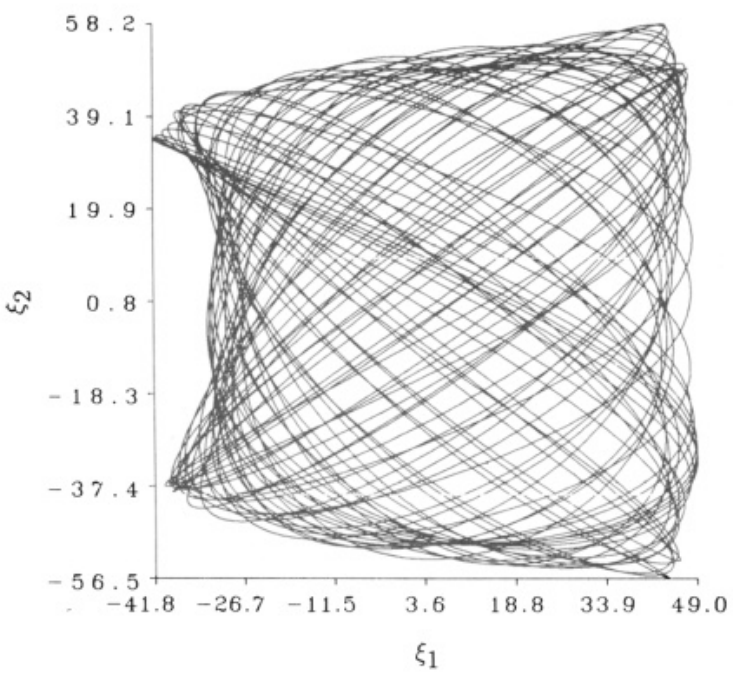

(a)

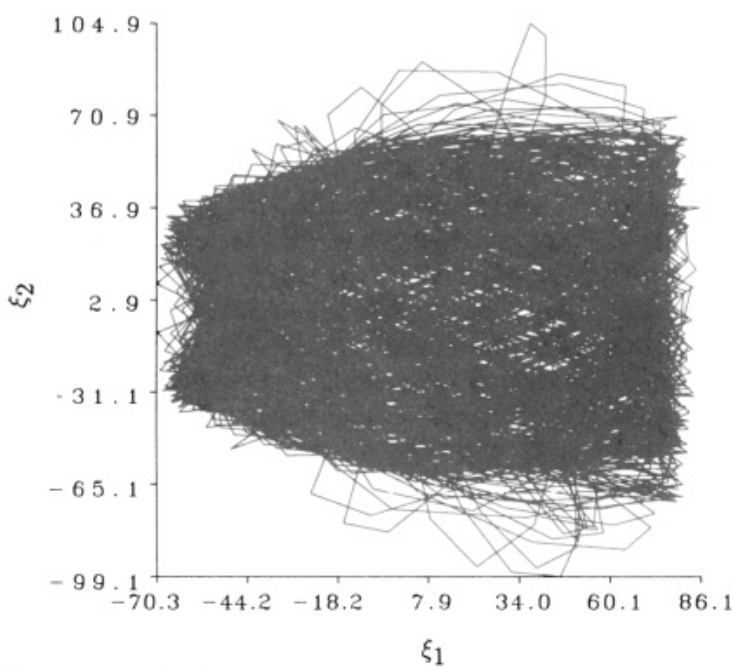

Figure 5. Projections onto the phase space plane $\left(\xi_{1}, \xi_{2}\right)$ of trajectories for $\mathrm{Na}_{3}$ with (a) molecule prepared in the $(0,0,0)$ vibrational state; (b) molecule in $(0.5,0.5,0.5)$.

known topographical features of the potential energy surface and were then expected. For the fundamental vibrational state (Figure 2) the spectra are essentially discrete, with well-defined absorption peaks that can be unambiguously assigned to the fundamental frequencies calculated in the harmonic approximation, namely $\omega_{1}=130 \mathrm{~cm}^{-1}, \omega_{2}=21 \mathrm{~cm}^{-1}$, and $\omega_{3}=106 \mathrm{~cm}^{-1}$. This type of spectra is indeed expected for trajectories initiated with an integral set of small vibrational quantum numbers. ${ }^{25}$ The occurrence of small additional absorption peaks in each spectrum is also noted.

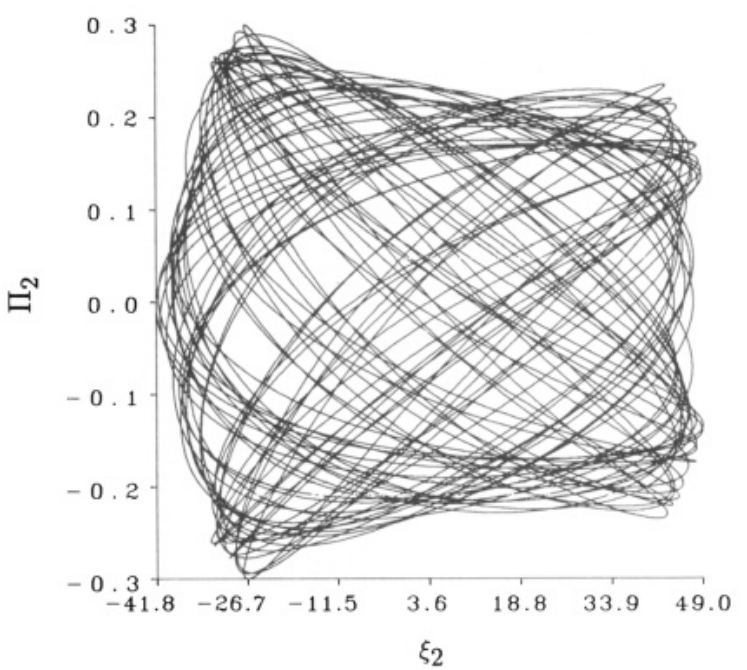

(a)

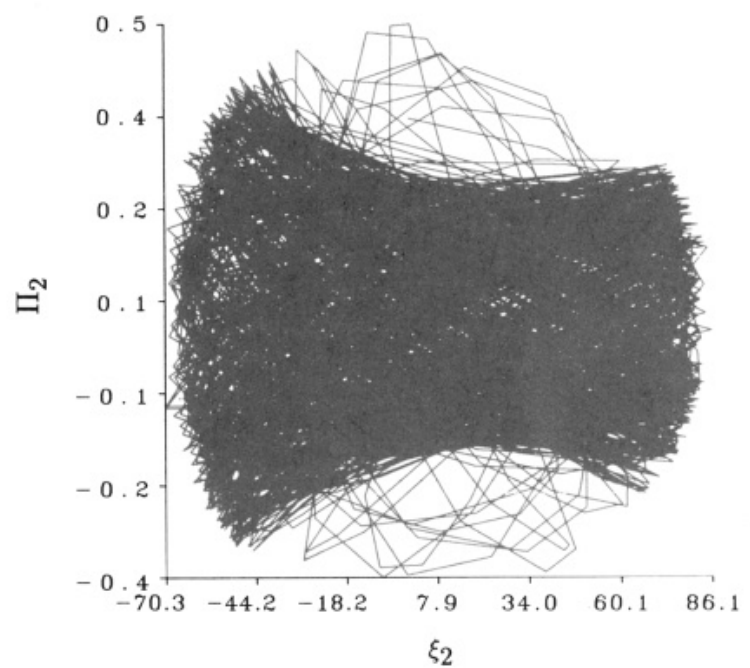

(b)

Figure 6. Same as in Figure 5 but for projections onto the phase space plane $\left(\xi_{2}, \Pi_{2}\right)$ : (a) molecule in $(0,0,0)$; (b) molecule in $(0.5,0.5,0.5)$.

We believe that these satellite peaks may be attributed to some reminiscent chaoticity even though one is dealing with very low energies.

On the other hand, for higher vibrational excitations (Figures 3 and 4), the spectra are no longer sharp rather becoming increasingly broadened and continuous. These features clearly reflect considerable deviations from the quasi-periodic regime. Nevertheless, although the line widths are quite large, we observe that peaks corresponding to different normal modes are clearly distinguishable.

The same behavior is apparent in Figures 5 and 6, which show plots of the projections of the trajectories onto selected space planes are displayed. The predominant quasi-periodic nature of the $(0,0,0)$ trajectory is clearly visible although, in a large time scale, a trend to uniformly fill up all the available phase space delimited by the caustics is also apparent. This behavior may be contrasted with the highly erratic motion of the trajectory for the $(0.5,0.5,0.5)$ vibrational state. Note that the jagged nature of the trajectories in Figure $5 \mathrm{~b}$ and Figure $6 \mathrm{~b}$ is simply due to the limited number of points used to make the plot, which is smaller than that employed for the Fourier analysis. The noise and line broadening in the power spectra are thus believed to be a genuine indication of the onset of chaotic behavior, and not a consequence of the sampling used for the Fourier analysis. In fact, the number of such sampled points has been selected as a power of 2 with the optimum exponent being chosen from the requirement that the spectral features were stable.

Two major conclusions may then be drawn from the above results regarding the intramolecular dynamics of $\mathrm{Na}_{3}$ : (i) chaoticity grows, as expected, with increasing internal energy; (ii) 
the nature of the vibrational motion at the zero-point vibrational energy is predominantly quasi-periodic, although some contamination of chaotic origin is already present. Thus, without more quantitative results, we cannot unambiguously characterize the unimolecular dynamics at this energy as being purely quasi-periodic.

4.2. Maximum Lyapunov Characteristic Numbers. For the estimation of the value of the maximal Lyapunov characteristic number $(\lambda)$ we have used the method described by Benettin et al., ${ }^{8}$ which involves running two parallel adjacent trajectories and periodically resetting their distance to the initial value. Accordingly, $\lambda$ has been estimated as

$$
\lambda=\lim _{n \rightarrow \infty} \frac{1}{n \tau} \sum_{i=1}^{n} \frac{\left|d_{i}\right|}{\left|d_{0}\right|}
$$

where $\left|d_{0}\right|$ is the initial separation between the two trajectories. The other $\left|d_{i}\right|$ are defined as follows: $\left|d_{1}\right|$ is the separation after a time $\tau$. The trajectories are slid a distance $\left|d_{0}\right|$ apart again and integrated a time $\tau$ to produce a separation $\left|d_{2}\right|$, and so on. The process is repeated until convergence of eq 15 , which Benettin et al. have shown to exist on the basis of previously proved theorems. Moreover, they have shown that the maximal Lyapunov characteristic number so obtained is independent of the time interval $\tau$ (provided it is small enough) and the initial conditions $\Delta \mathbf{q}$ and $\Delta p$. In addition, numerical calculations on model potentials have shown that $\lambda$ is zero for quasi-periodic trajectories and is a positive number for stochastic trajectories. In this case, $\lambda$ may assume different (positive) values for trajectories initiated in regions of phase space with different ergodic components. ${ }^{11}$ Thus $\lambda$ becomes a function of the total energy, and is essentially a global measure of local exponential instability.

In physical terms the inverse of the maximal Lyapunov characteristic number has the dimension of a time, and may be intepreted as a characteristic time of the system to be compared with the periods of the vibrational motion. If the latter are of the same order of magnitude of $\lambda$, the phase of the vibration is subject to a random modulation giving rise to a broadening of the spectral lines. ${ }^{26,27}$ More precisely, the maximal Lyapunov characteristic number represents an average rate with which two initially infinitesimally close trajectories separate and is characteristic of the memory of the system. In this work we have chosen the number of points at which the two trajectories were reset to be $10^{5}$, a value which proved to be large enough to achieve a fast and uniform convergence of the results to the asymptotic limit.

Because the trajectories may be highly unstable, accuracy in the numerical integrations play a critical role. For this purpose we have used a fifth-order Runge-Kutta method with a time step of $7.8125 \times 10^{-12} \mathrm{ps}$, each trajectory being propagated over a time interval of $5 \times 10^{-10} \mathrm{ps}$. Each primary trajectory has been started at the equilibrium geometry of the ${ }^{2} \mathrm{~B}_{2}$ minimum on the $\mathrm{Na}_{3}$ potential energy surface, with the kinetic energy being equally distributed among the vibrational degrees of freedom. Classical states varying from $(-0.49,-0.49,-0.49)$ to $(5.0,5.0,5.0)$ have been considered, which cover the energy range $0.0308 \mathrm{~kJ} \mathrm{~mol}^{-1} \leq E$ $\leq 16.94 \mathrm{~kJ} \mathrm{~mol}^{-1}$. The initial (Euclidian) distance between the two trajectories was typically $10^{-7}$ au in the direction of the momentum $P_{1}$. To test the reliability of the results from the present work, some calculations were also made with the parallel trajectory displaced along a different direction in phase space. For further testing purposes, a calculation was repeated using 2 $\times 10^{5}$ resetting steps. No significant differences have been found.

The calculations have been carried out on a CDC 4680 computer using double precision (word size $=64$ bits), having some of these calculations been repeated for a higher numerical precision (120 bits) on a CDC Cyber 830 . This increase in precision resulted in a faster convergence of the estimates for the maximal Lyapunov characteristic number. Because such calculations are very time consuming (a typical orbit takes ca. $10^{4} \mathrm{~s}$ of CPU time on the CDC 4680 computer), we cannot claim having done a very exhaustive analysis.

Figure 7 illustrates a typical behavior of the maximal Lyapunov characteristic number for the $(1,1,1)$ vibrational state of $\mathrm{Na}_{3}$. The

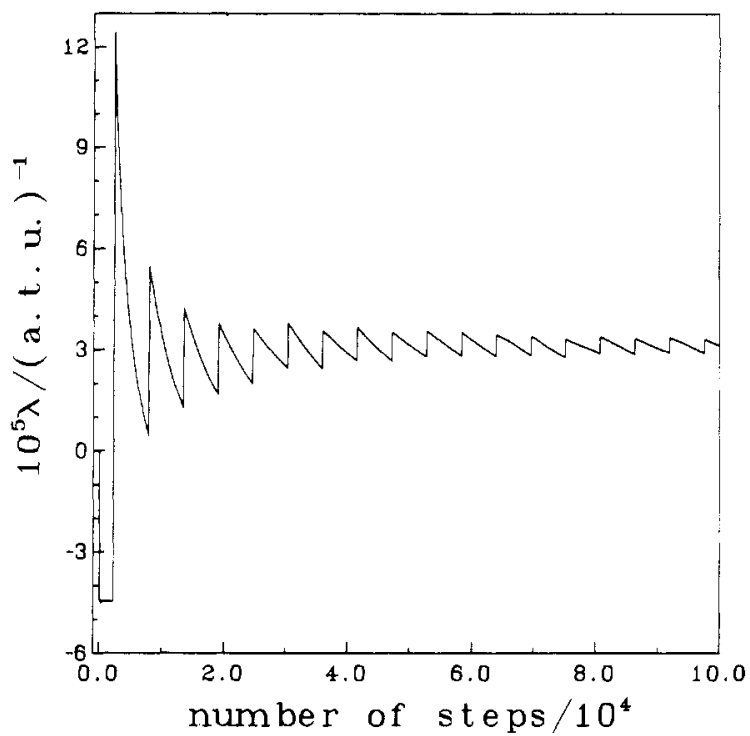

Figure 7. Plot of the maximal Lyapunov characteristic number $(\lambda)$ as a function of the number of calculation steps for the $(1,1,1)$ vibrational state of $\mathrm{Na}_{3}$.

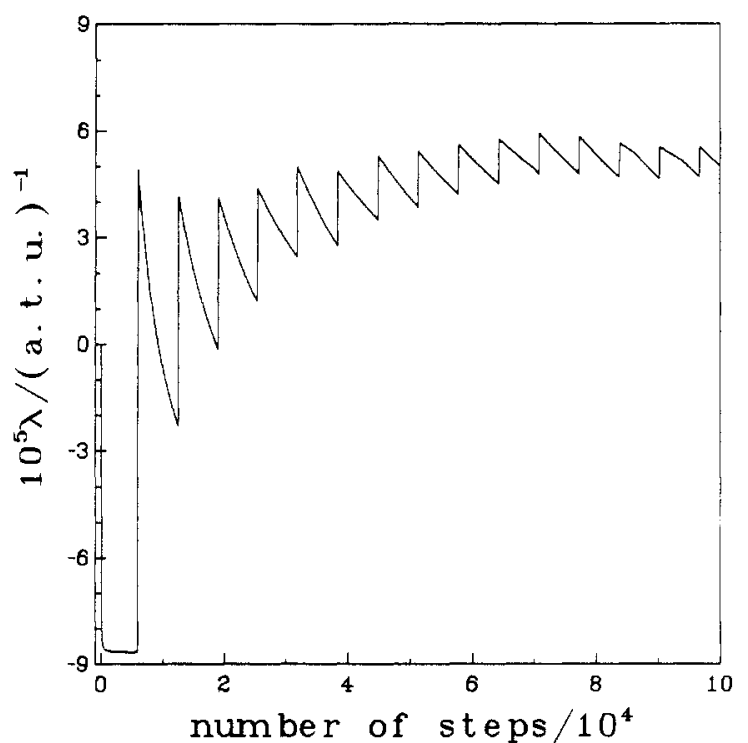

Figure 8. Same as in Figure 7 but for the $(4,4,4)$ vibrational state of $\mathrm{Na}_{3}$.

convergence of the estimate toward a small but nonzero limiting value is clearly seen to be rather fast. Figure 8 illustrates a similar plot but for an higher excited vibrational state of $\mathrm{Na}_{3}$, i.e., $(4,4,4)$. As for the $(1,1,1)$ state, the convergence is fast but tending now to a somewhat larger asymptotic value, which is a clear indication of the stochastic nature of the dynamics at this energy.

The global behavior of the maximal Lyapunov characteristic number as a function of the energy is shown in Figure 9. As expected from theoretical grounds, the maximal Lyapunov characteristic number raises almost uniformly with increasing energy reaching a nearly constant value of ca. $5 \times 10^{-5} \mathrm{atu}^{-1}$ before dissociation into an atom plus a diatom, which occurs at an energy of $26.5 \mathrm{~kJ} \mathrm{~mol}^{-1}$ [i.e., close to the $(8,8,8)$ vibrational state]. Two perturbations to this general trend of increasing monotonic behavior should be pointed out though. One takes place at an energy of ca. $2 \mathrm{~kJ} \mathrm{~mol}^{-1}$, i.e. between the $(0.25,0.25,0.25)$ and $(0.3,0.3,0.3)$ classical levels. The other takes place at ca. $9 \mathrm{~kJ} \mathrm{~mol}^{-1}$, i.e. between $(2.5,2.5,2.5)$ and $(2.7,2.7,2.7)$. A possible rationalization for these abrupt changes consists in relating them to the energy barriers that the system must overcome (see Figure 1): $0.986 \mathrm{~kJ} \mathrm{~mol}^{-1}$ (or, in terms of the continuous variable $n=n_{1}=n_{2}=n_{3}, n=$ -0.18 ) to pass from the equilibrium ${ }^{2} B_{2}$ structure (absolute minimum) to linear $\left({ }^{2} \Sigma_{u}^{+}\right), 4.558 \mathrm{~kJ} \mathrm{~mol}^{-1}(n=0.98)$ to pass from ${ }^{2} \mathrm{~B}_{2}$ to acute" $\left({ }^{2} \mathrm{~A}_{1}\right)$ geometries, and $15.585 \mathrm{~kJ} \mathrm{~mol}^{-1}(n=4.56)$ 
Exponentiating Trajectories

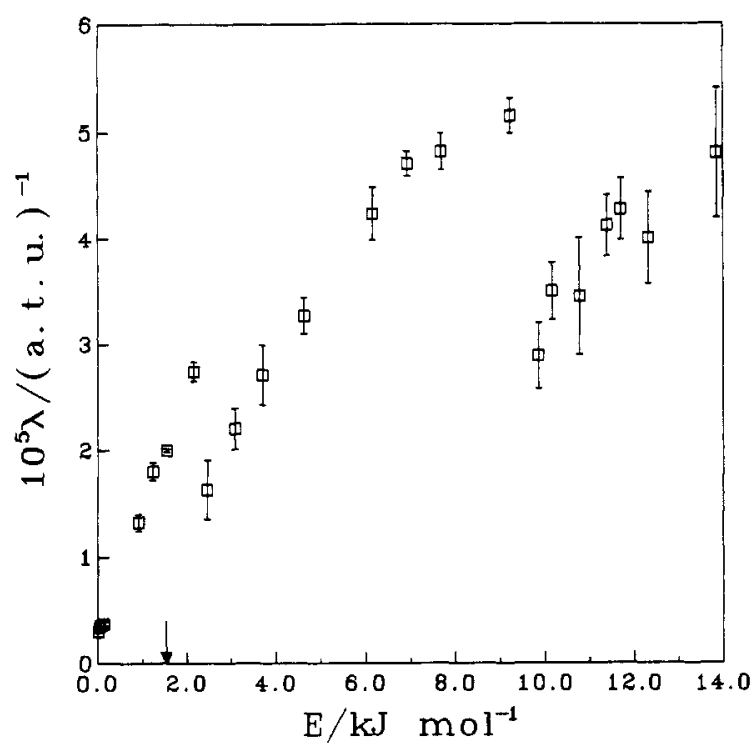

Figure 9. Plot of the calculated maximal Lyapunov characteristic number of $\mathrm{Na}_{3}$ as a function of the internal energy. The arrow indicates the zero-point energy.

for reaching the minimum-energy $D_{3 h}$ structure $\left({ }^{2} \mathrm{E}^{\prime}\right)$. Indeed, one expects a sudden increase in the accessible volume of phase space at such critical energies. However, to fill up the whole available phase space the trajectory must overpass bottlenecks. As a result the phase space sampled by the trajectory may diminish in relative terms if it fails to overpass those bottlenecks due to any dynamical restrictions, which could explain the momentary lowering of the maximal Lyapunov exponent. Several chaotic components may therefore become important in characterizing the true intramolecular dynamics. A word of caution is, however, appropriate at this point. Indeed, although unlikely, the possibility that the discontinuities observed in Figure 9 may partly be due to an insufficient convergence of the calculation or to the restricted number of trajectories sampled cannot be entirely ruled out. Moreover we cannot ensure that the discontinuities in Figure 9 are, at least in part, not due to the specific (although commonly used $^{7}$ ) sampling procedure chosen for starting the trajectories and to the limited time of integration. Of course, if the trajectories were integrated over an infinite period of time then the strategy for sampling would become irrelevant given the possibility of energy randomization between the various degrees of freedom. In summary, a clear answer to whether the discontinuities in Figure 9 are a real physical effect may require further investigations on this and similar systems.

Near the zero-point vibrational energy, and even at energies very close to the classical minimum of the potential energy surface, the maximal Lyapunov characteristic number is already nonzero and clearly positive. This suggests a very early onset of the chaotic regime for this dynamical system. Such early onset of chaos has already been noted in other floppy systems such as KCN. ${ }^{28}$

The inverse of the average maximal Lyapunov characteristic number is a characteristic time for the randomization of the energy in the molecule. This relaxation time can be estimated from the results shown in Figure 9 to be ca. $2 \times 10^{4}$ atu, which is of the order of two harmonic stretching vibration periods. In this time interval internal energy has been completely randomized among the internal degrees of freedom, which supports the conjecture of a very rapid energy relaxation. Thus, one expects statistical features to primarily determine the dynamics rather than dynamical ones. This result supports previous findings on atomdiatom reactive scattering for analogous systems (namely, $\mathrm{Li}+$ $\mathrm{Li}_{2} \rightarrow \mathrm{Li}_{2}+\mathrm{Li},{ }^{29} \mathrm{Li}+\mathrm{Na}_{2} \rightarrow \mathrm{LiNa}+\mathrm{Na},{ }^{30}$ and $\mathrm{Na}+\mathrm{K}_{2} \rightarrow$ $\mathrm{NaK}+\mathrm{K}^{30}$ ), which have indicated a high degree of statistical behavior in the corresponding dynamics.
The Journal of Physical Chemistry, Vol. 96, No. 14, 19925709

\section{Conclusions}

We have carried out an intramolecular dynamics study for the sodium trimer using a realistic potential energy surface. The results of the present calculations seem to support the conjecture that a very fast redistribution of the internal energy occurs between the internal degrees of freedom. In fact the memory of the system has been found to be characterized by a rather small relaxation time. This is the time scale on which the details of the dynamics are expected to be important. It may then be argued that a statistical mechanics description of the system should be valid for most energies of interest.

However, we have also observed a sharp nature in the power spectra of the sodium trimer at very low energies. This characteristic is compatible with a predominantly quasi-periodic regime at those energies. Although apparently contradictory, the observation of these two regimes may indeed help to rationalize the hybrid direct-statistical type of behavior which was found in atom-diatom reaction dynamics studies ${ }^{29,30}$ involving the analogous $\mathrm{Li}_{3}, \mathrm{LiNa}_{2}$, and $\mathrm{NaK}_{2}$ trimeric species. It would be interesting if the present findings could be confirmed from further dynamics calculations on these systems, possibly based on different criteria to predict the onset of the chaotic behavior and on energy distributions which bear more resemblance to those which are observed in the reactive scattering experiments.

Acknowledgment. This work was supported by the Instituto Nacional de Investigação Cientifica (INIC), Portugal. The allocation of computer time at the University of Oporto is also gratefully acknowledged.

Registry No. $\mathrm{Na}_{3}, 37279-42-8$.

\section{References and Notes}

(1) Robinson, P. J.; Holbrook K. A. Unimolecular Reactions; Wiley: New York, 1972

(2) Forst, W. Theory of Unimolecular Reactions; Academic Press: New York, 1973.

(3) Sinai, Ya. G. Introduction to Ergodic Theory; Princeton University Press: Princeton, 1976.

(4) Arnold, V. I. Ergodic Problems in Classical Mechanics; Benjamin: New York, 1973.

(5) Krylov, N. S. Works on the Foundations of Statistical Physics; Princeton University Press: Princeton, 1979.

(6) Hansel, K. D. Chem. Phys. 1978, 33, 35

(7) Farantos, S. C.; Murrell, J. N. Chem. Phys. 1981, 55, 205.

(8) Benettin, G.; Galgani, L.; Strelcyn, J.-M. Phys. Rev. 1976, A14, 2338. (9)

(10) Ford, J. Adv. Chem. Phys, 1973, 24, 155

(11) Contopoulos, G.; Galgani, L.; Giorgilli, A. Phys. Rev. 1978, Al8, 1183.

(12) Galgani, L.; Scotti, A. Riv. Nuovo Cimento 1972, 2, 189

(13) Ford, J.; Stoddard, S. D.; Turner, J. S. Progr. Theor. Phys. 1973, 50, 1547.

(14) Casati, G.; Ford, J. Phys. Rev. 1979, A12, 1702.

(15) Varandas, A. J. C.: Morais, V. M. F. Mol. Phys. 1982, 47, 1241

(16) Cross, P. C.; van Vleck, J. H. J. Chem. Phys. 1933, $I, 350$.

(17) Bunker, D. L. J. Chem. Phys. 1962, 37, 393.

(18) Wilson Jr., E. B.; Decius, J. C.; Cross, P. C. Molecular Vibrations; McGraw-Hill: New York, 1955.

(19) Herzberg, G. Molecular Spectra and Molecular Structure; van Nostrand: New York, 1964; Vol. II, p 148.

(20) Varandas, A. J. C. Chem. Phys. Lett. 1987, 138, 455.

(21) Muckerman, J. T. Quantum Chemistry Program Exchange; Indiana University: Bloomington, IN, 1973; no. 229.

(22) Varandas, A. J. C.; Morais, V. M. F.; Pais, A. A. C. C. Mol. Phys. $1986,58,285$.

(23) Subroutine FTFPS, International Mathematical and Statistical Libraries.

(24) Cooley, J. M.; Turkey, J. W. Math. Comp. 1965, 19, 297.

(25) Noid, D. W.; Koszykowski, M. L.; Marcus, R. A. J. Chem. Phys. $1977,67,404$

(26) Kubo, R. Adv. Chem. Phys. 1969, 15, 101.

(27) Kubo, R. Lect. Theoret. Phys. 1959, 1, 181.

(28) Tennyson, J.; Farantos, S. C. Chem. Phys. Lett. 1984, 109, 160.

(29) Morais, V. M. F.; Varandas, A. J. C. J. Chem. Soc., Faraday Trans. 2 1989, 85, 1 .

(30) Morais, V. M. F.; Varandas, A. J. C. J. Chem. Soc., Faraday Trans. $21987,83,2247$. 